\title{
Efficient Signature Schemes Based on Birational Permutations
}

\author{
Adi Shamir \\ Dept. Computer Science, The Weizmann Institute of Science, Rehovot 76100, Israel
}

\begin{abstract}
Many public key cryptographic schemes (such as cubic RSA) are based on low degree polynomials whose inverses are high degree polynomials. These functions are very easy to compute but time consuming to invert even by their legitimate users. To overcome this problem, it is natural to consider the class of birational permutations $f$ over k-tuples of numbers, in which both $f$ and $f^{-1}$ are low degree rational functions. In this paper we develop two new families of birational permutations, and discuss their cryptographic applications.
\end{abstract}

Remark: At the rump session of CRYPTO 93, Coppersmith Stern and Vaudenay presented two elegant and powerful attacks on the two signature schemes suggested in this paper. The attacks are quite specific, and thus it is conceivable that other signature schemes based on birational permutations will not be affected. The reader is thus encouraged to study the underlying mathematical structure of the schemes and the attacks, but to excercise great caution in implementing the ideas in practice.

\section{Introduction}

The original proposal for public key cryptography (Diffie and Hellman [1976] was based on the notion of trapdoor permutations, i.e., invertible functions which are easy to compute but apparently difficult to invert, unless some trapdoor information (which makes the inversion easy) is known. The best known implementation of this idea is the RSA scheme (Rivest, Shamir and Adleman [1978]), which can solve in a unified way the problems of key management, secure transmission, user identification, message authentication, and digital signatures. In one of the variants of this scheme, the encryption function is the low degree polynomial $f(x)=x^{3}(\bmod n)$, which can be efficiently computed with two 
modular multiplications. Unfortunately, its inverse $f^{-1}(v)=v^{d}(\bmod n)$ is a very high degree polynomial, and thus its evaluation is quite slow (especially in software implementations).

In spite of extensive research in the last 16 years, there had been no fundamentally new constructions of trapdoor permutations which are faster than the RSA scheme. To overcome this difficulty, researchers have developed specialized solutions to various cryptographic needs which are not based on this unifying notion. For example, Diffie and Hellman [1976] proposed a key management scheme which is based on the one-way permutation of exponentiation modulo a prime. Since this function cannot be efficiently inverted, it is neither an encryption nor a signature scheme. The cryptosystem of Merkle and Hellman [1978] is invertible, but its mapping is not onto and thus it can not generate digital signatures. The Fiat-Shamir [1986] and DSS [1991] signature schemes are not one-to-one mappings, and thus they can not be used as cryptosystems.

A natural approach to this problem is to search for low degree algebraic mappings (polynomials or rational functions) whose inverses are also low degree algebraic mappings. Such mappings are called birational functions. We are particularly interested in multivariate mappings $f\left(x_{1}, \ldots, x_{k}\right)=\left(v_{1}, \ldots, v_{k}\right)$ in which the $x_{i}$ and the $v_{i}$ are numbers modulo a large $n=p q$, since the solution of general algebraic equations of this type is at least as hard as the factorization of the modulus. In this context, we say that a polynomial modulo $n$ is low degree if its degree is a constant which does not grow with $n$, and a rational function is low degree if it is the ratio of two low degree polynomials. For example, in the case of cubic RSA, the function is considered low degree, but its inverse is not. Nonlinear algebraic mappings do not usually have unique inverses, when they do have inverses they usually cannot be written in closed form, and when the closed forms exist they are usually based on root extractions (radicals) or exponentiations whose computation modulo a large $n$ is very slow. The construction of nonlinear birational mappings is thus a non-trivial task.

One attempt to construct birational permutations, due to Fell and Diffie [85], used the following DES-like idea. Let $\left(x_{1}, x_{2}, \ldots, x_{k}\right)$ be an initial $k$-vector of variables, and let $g$ be a secret nonlinear multivariate polynomial. Alternately replace the current $k$-vector of multivariate polynomials $\left(p_{1}, p_{2}, \ldots, p_{k}\right)$ by $\left(p_{1}+g\left(p_{2}, \ldots, p_{k}\right), p_{2}, \ldots, p_{k}\right)$, and rotate the $k$-vector to the right. After sufficiently many iterations, expand and publish the resultant $k$-vector of multivariate polynomials as your public key. When the trapdoor information $g$ is known, the inverse of $f$ can be computed by undoing the transformations (i.e., by alternately subtracting $p_{1}$ and rotating the $k$-vector to the left). Unfortunately, even when $g$ is a quadratic function, the degree (and thus the size of the public key) grows exponentially with the number of iterations, which cannot be too small for security reasons. As the authors themselves conclude, "there seems 
to be no way to build such a system that is both secure and has a public key of practical size".

The problem is not accidental, due to the following generic attack: If $f$ is known, the cryptanalyst can prepare a large number of input-output pairs for this function. Since $f$ is invertible, these pairs (in reverse order) can be used to interpolate the unknown low-degree function $f^{-1}$ by solving a small number of linear equations relating its coefficients. We do not know how to solve this problem in the context of public key cryptosystems. However, in the context of public key signature schemes there is a simple way to avoid this specific attack with the following modification:

Key Generation: Each user in the system chooses a particular birational permutation $f\left(x_{1}, \ldots, x_{k}\right)=\left(v_{1}, \ldots, v_{k}\right)$ consisting of $k$ rational functions $f_{i}\left(x_{1}, \ldots\right.$, $\left.x_{k}\right)=v_{i}$, discards the first $s>0$ of these $f_{i}$ functions, describes the other $k-s$ $f_{i}$ functions in his public key, and keeps the inverse of $f$ as his private key.

Signature Generation: Given a digital message $m$, the signer chooses $v_{i}=r_{i}$ for $i=1, \ldots, s$, and computes $v_{i}=h(m, i)$ for $i=s+1, \ldots, k$, where $r_{i}$ are newly chosen secret random values, and $h$ is a publicly known cryptographic hash function. He then uses his knowledge of the secret $f^{-1}$ to compute a signature $\left(x_{1}, \ldots, x_{k}\right)$ satisfying $f\left(x_{1}, \ldots, x_{k}\right)=\left(v_{1}, \ldots, v_{k}\right)$.

Signature Verification: The verifier checks that $f_{i}\left(x_{1}, \ldots, x_{k}\right)=h(m, i)$ for $i=s+1, \ldots, k$, where the $f_{i}$ 's are taken from the signer's public key.

This modified scheme can no longer be used as a cryptosystem, since the cleartext $\left(x_{1}, \ldots, x_{k}\right)$ cannot be uniquely recovered from the shorter ciphertext $\left(v_{s+1}, \ldots\right.$, $v_{k}$ ). It can be used as a signature scheme, since messages can have multiple signatures. The cryptanalyst cannot interpolate $f^{-1}$ since it is not uniquely defined by the public key: He cannot generate complete input-output pairs for $f^{-1}$ by himself, and cannot use input-output pairs generated by the legitimate signer since each one of them is based on new unknown values $r_{i}$. The recommended choice of $s$ is 1 , which makes the verification condition hardest to satisfy.

The security of this scheme depends on the choice of birational permutations. In Section 2 we introduce a simple family of birational permutations based on sequentially linearized equations, and in Section 3 we introduce a more sophisticated family of birational permutations based on algebraic bases in polynomial rings. Both families yield signature schemes with very low computational complexity. Unfortunately, both families also turn out to be breakable by the new attacks of Coppersmith Stern and Vaudenay[1993]. The reader is encouraged to look for other families of birational permutations and to study their efficiency and security. 


\section{A Birational Permutation Based on Sequentially Lin- earized Equations}

Let $n$ be the public product of two large secret primes $p$ and $q$. verify Consider the triangular birational permutation $g\left(y_{1}, \ldots, y_{k}\right)=\left(w_{1}, \ldots, w_{k}\right)(\bmod$ $n$ ) in which the $i$-th output depends only on the first $i$ inputs via the mapping $g_{i}\left(y_{1}, \ldots, y_{i}\right)=w_{i} \quad(\bmod n)$, where $g_{i}$ is a low degree polynomial which is linear in its last input $y_{i}$ (the other inputs can and should occur non-linearly). Given the values of inputs $y_{1}, \ldots, y_{k}$, we can easily compute the values of the outputs $w_{1}, \ldots, w_{k}$ by evaluating $k$ low degree polynomials. Given the values of the outputs $w_{1}, \ldots, w_{k}$, we can easily recover the values of the inputs $y_{1}, \ldots, y_{k}$ by solving a series of linear equations: First we solve for $y_{1}$ in $g_{1}\left(y_{1}\right)=w_{1}(\bmod n)$. Then we substitute the computed value of $y_{1}$ into its (non-linear) occurrences in $g_{2}\left(y_{1}, y_{2}\right)=w_{2}(\bmod n)$, and solve the remaining linear equation in $y_{2}$. We proceed in this order until we compute the last $y_{k}$. Each $y_{i}$ is thus a low degree rational function of the $w_{j}$ 's, which is easy to compute with a small number of arithmetic operations modulo $n$.

To hide the easy solvability of the $g_{i}$ 's, the user has to transform them into more random looking polynomials before publishing them as his public key. We recommend the following two transformations:

1. Let $A$ be a randomly chosen invertible $k \times k$ matrix, and consider the variable transformation $Y=A X$, where $Y$ is the column vector of original variables $\left(y_{1}, \ldots, y_{k}\right)^{t}$ and $X$ is a column vector of new variables $\left(x_{1}, \ldots, x_{k}\right)^{t}$. When the resultant polynomials are expanded, they contain all the variables in a non-linear way.

2. Let $B$ be a randomly chosen invertible $k \times k$ matrix, and consider the mixing transformation $F=B G$, where $G$ is the column vector of polynomials $\left(g_{1}, \ldots, g_{k}\right)^{t}$ and $F$ is a column vector of new polynomials $\left(f_{1}, \ldots, f_{k}\right)^{t}$. Each $f_{i}$ is thus a polynomial whose coefficients are random linear combinations of the corresponding coefficients of the given $g_{1}, \ldots, g_{k}$.

When $A$ and $B$ are known, it is easy to solve the resultant system of equations $f_{i}\left(x_{1}, \ldots, x_{k}\right)=v_{i}(\bmod n)$ for $i=1, \ldots, k$ by inverting these transformations. of them change be To minimize the size of the public key, we recommend using $g_{i}$ 's which are homogeneous quadratic expressions of the form:

$$
g_{i}\left(y_{1}, \ldots, y_{i}\right)=l_{i}\left(y_{1}, \ldots, y_{i-1}\right) \cdot y_{i}+q_{i}\left(y_{1}, \ldots, y_{i-1}\right) \quad(\bmod n)
$$

where $l_{i}$ is a randomly chosen linear function of its inputs and $q_{i}$ is a randomly chosen homogencous quadratic function of its inputs. The only exception is $g_{1}$ in which $l_{1}$ and $q_{1}$ have no inputs. Since the coefficients of the linear $g_{1}$ cannot 
be mixed with the coefficients of the quadratic $g_{2}, \ldots, g_{k}$, and since we have to eliminate at least one of the polynomials in order to overcome the interpolation attack, we recommend the elimination of $g_{1}$ from the user's public key.

Without loss of generality, we can assume that $g_{1}\left(y_{1}\right)=y_{1}$ and $g_{2}\left(y_{1}, y_{2}\right)=$ $y_{1} y_{2}$ (since they can always be brought to this form by linear transformations). The case $k=2$ is thus equivalent to the OSS scheme (Ong, Schnorr, Shamir [STOC83]), where the variable transformation $A$ is $y_{1}=x_{1}+a x_{2}, y_{2}=x_{1}-a x_{2}$ and the mixing transformation $B$ is the identity (all the arithmetic operations are carried out modulo a composite $n$ ). solves the signature The OSS scheme was successfully attacked by Pollard [1984], who showed that one quadratic equation in two variables can be solved even when the factorization of the modulus is unknown. A typical example of the extended signature scheme for $k=3$ with the toy modulus $n=101$ is:

Example: Consider the following sequentially linearized system of equations:

$$
\begin{aligned}
y_{1} & =w_{1} \quad(\bmod 101) \\
y_{1} y_{2} & =w_{2}(\bmod 101) \\
\left(29 y_{1}+43 y_{2}\right) y_{3} & +\left(71 y_{1}^{2}+53 y_{2}^{2}+89 y_{1} y_{2}\right)=w_{3} \quad(\bmod 101)
\end{aligned}
$$

Apply the linear change of variables:

$$
\begin{array}{ll}
y_{1}=x_{1}+25 x_{2}+73 x_{3} & (\bmod 101) \\
y_{2}=x_{1}+47 x_{2}+11 x_{3} & (\bmod 101) \\
y_{3}=x_{1}+83 x_{2}+17 x_{3} & (\bmod 101)
\end{array}
$$

to obtain the new expressions:

$$
\begin{array}{cc}
x_{1}+25 x_{2}+73 x_{3}=w_{1} \quad(\bmod 101) & \\
x_{1}^{2}+64 x_{2}^{2}+96 x_{3}^{2}+72 x_{1} x_{2}+84 x_{1} x_{3}+70 x_{2} x_{3}=w_{2} & (\bmod 101) \\
83 x_{1}^{2}+55 x_{2}^{2}+16 x_{3}^{2}+28 x_{1} x_{2}+97 x_{1} x_{3}+74 x_{2} x_{3}=w_{3} & (\bmod 101) .
\end{array}
$$

Mix these three expressions $g_{1}, g_{2}$, and $g_{3}$ by computing: $f_{1}=g_{1}(\bmod 101)$, $f_{2}=\left(39 g_{2}+82 g 3\right) \quad(\bmod 101), f_{3}=\left(93 g_{2}+51 g 3\right) \quad(\bmod 101)$. The resultant expressions are:

$$
\begin{aligned}
x_{1}+25 x_{2}+73 x_{3}=v_{1} & (\bmod 101) \\
78 x_{1}^{2}+37 x_{2}^{2}+6 x_{3}^{2}+54 x_{1} x_{2}+19 x_{1} x_{3}+11 x_{2} x_{3}=v_{2} & (\bmod 101) \\
84 x_{1}^{2}+71 x_{2}^{2}+48 x_{3}^{2}+44 x_{1} x_{2}+33 x_{1} x_{3}+83 x_{2} x_{3}=v_{3} & (\bmod 101)
\end{aligned}
$$


Discard the first equation, publish the last two equations as your public key, and retain the variable transformation $\mathrm{A}$ and the mixing transformation $\mathrm{B}$ as your secret key.

To sign a message $m$, hash it into two numbers $v_{2}, v_{3}$ in $[0,101)$. Assume that $v_{2}=h(m, 2)=12$ and $v_{3}=h(m, 3)=34$. Its signature is any triplet of numbers $x_{1}, x_{2}, x_{3}$ which satisfies the two published equations for these values of $v_{2}, v_{3}$. To find such a solution, choose a random value for $v_{1}$ (each choice will yield a different signature). For example, let $v_{1}=99$. Apply the inverse mixing transformation $B^{-1}$ by computing $w_{1}=v_{1}=99(\bmod 101), w_{2}=8 v_{2}+v_{3}=$ $29(\bmod 101), w_{3}=27 v_{2}+18 v_{3}=27 \quad(\bmod 101)$. The equations in terms of the $y_{i}$ 's are thus:

$$
\begin{aligned}
y_{1} & =99 \quad(\bmod 101) \\
y_{1} y_{2} & =29 \quad(\bmod 101) \\
\left(29 y_{1}+43 y_{2}\right) y_{3} & +\left(71 y_{1}^{2}+53 y_{2}^{2}+89 y_{1} y_{2}\right)=27 \quad(\bmod 101)
\end{aligned}
$$

To solve it, substitute $y_{1}=99$ into the second equation and solve the linear equation $99 y_{2}=29(\bmod 101)$ to obtain $y_{2}=36$. Substitute both values into the third equation, simplify it into $76 y_{3}+45=27(\bmod 101)$, and solve it to obtain $y_{3}=29$. Finally, use the inverse of the variable transformation $A$ to change this $Y$ solution into the $X$ solution $x_{1}=40, x_{2}=27, x_{3}=22$ which is a valid signature of $m$ since it satisfies the two published equations.

Two general homogeneous quadratic equations in three unknowns have 12 coefficients, but they can be changed without loss of generality into a normal form which reduces the size of the public key to five numbers. The size of each signature is 196 bytes (when $n$ is a 512-bit modulus). The generation and verification of each signature requires about 20 modular multiplications, compared to about 760 modular multiplications in the RSA scheme and about 50 modular multiplications in the Fiat-Shamir Scheme.

\section{A Birational Permutation Based On Algebraic Bases}

In this section we introduce a different family of birational permutations, which is inherently non-linear in all its components. The construction is quite unexpected, and seems to have many possible extensions and modifications.

Let $F_{d}\left[y_{1}, y_{2}, \ldots, y_{k}\right]$ denote the set of all the homogeneous polynomials of degree $d$ in the $k$ variables $y_{1}, y_{2}, \ldots, y_{k}$. We consider in particular the case of quadratic polynomials $(d=2)$, which are linear combinations of the $k(k+1) / 2$ elementary 
quadratics $y_{i} y_{j}$ for $i \leq j$ with coefficients $a_{i j}$. Such a set of quadratics is called a linear basis of $F_{2}\left[y_{1}, \ldots, y_{k}\right]$.

If we allow the additional operations of multiplication and (remainder-free) division of polynomials, we can express some of the elementary quadratics by other elementary quadratics. For example, the standard linear basis of $F_{2}\left[y_{1}, y_{2}, y_{3}\right]$ is $\left\{y_{1}^{2}, y_{2}^{2}, y_{3}^{2}, y_{1} y_{2}, y_{1} y_{3}, y_{2} y_{3}\right\}$. However, the first three elementary quadratics can be expressed by the last three in the following way:

$y_{1}^{2}=\left(y_{1} y_{2}\right)\left(y_{1} y_{3}\right) /\left(y_{2} y_{3}\right), y_{2}^{2}=\left(y_{1} y_{2}\right)\left(y_{2} y_{3}\right) /\left(y_{1} y_{3}\right), y_{3}^{2}=\left(y_{1} y_{3}\right)\left(y_{2} y_{3}\right) /\left(y_{1} y_{2}\right)$.

We can thus reduce the six linear generators $\left\{y_{1}^{2}, y_{2}^{2}, y_{3}^{2}, y_{1} y_{2}, y_{1} y_{3}, y_{2} y_{3}\right\}$ into the three algebraic generators $\left\{y_{1} y_{2}, y_{1} y_{3}, y_{2} y_{3}\right\}$. Another triplet of algebraic generators is $\left\{y_{1}^{2}, y_{1} y_{2}, y_{2} y_{3}\right\}$. However, $\left\{y_{1}^{2}, y_{2}^{2}, y_{2} y_{3}\right\}$ does not algebraically generate $F_{2}\left[y_{1}, y_{2}, y_{3}\right]$ since it cannot express $y_{1} y_{2}$.

To formalize this notion, consider an arbitrary set $G$ of polynomials over a ring $R$. Its algebraic closure $[G]$ of $G$ is defined as the smallest set of polynomials which contains $G$ and $R$, and is closed under addition, subtraction, multiplication, and remainder-free division. Note that $[G]$ is not necessarily an ideal in the ring of polynomials over $R$, since we do not allow arbitrary polynomials as coefficients of the generators. For example, when $G=\left\{y^{2}\right\},[G]$ is the set of all the polynomials in $y$ whose monomials have even degrees. Note further that root extractions (radicals) are not allowed as basic operations, since they cannot be carried out efficiently in some rings $R$. $G$ is called an algebraic basis of $F$ if the polynomials in $G$ are algebraically independent and $F$ is contained in $[G]$.

Theorem: 1 . The set of polynomials $\left\{y_{1}^{2}, y_{1} y_{2}, y_{2} y_{3}, \ldots, y_{k-1} y_{k}\right\}$ is an algebraic basis of $F_{2}\left[y_{1}, y_{2}, \ldots, y_{k}\right]$ for any $k$.

2. The set of polynomials $\left\{y_{1} y_{2}, y_{2} y_{3}, y_{3} y_{4}, \ldots, y_{k} y_{1}\right\}$ is an algebraic basis of $F_{2}\left[y_{1}, y_{2}, \ldots y_{k}\right]$ for any odd $k>1$.

Proof (sketch): We show that the specified sets can generate all the other elementary quadratics $y_{i} y_{j}$ for $i \leq j$. Assume first that $j-i$ is an odd number. Then we can use the telescoping formula:

$$
y_{i} y_{j}=\left(y_{i} y_{i+1}\right)\left(y_{i+2} y_{i+3}\right) \ldots\left(y_{j-1} y_{j}\right) /\left(y_{i+1} y_{i+2}\right) \ldots\left(y_{j-2} y_{j-1}\right) \text {. }
$$

If $j-i$ is an even number, this approach will yield $y_{i} / y_{j}$ instead of $y_{i} y_{j}$. To turn the former into the later, we have to multiply it by $y_{j}^{2}$. In case 1 , we are given $y_{1}^{2}$ as a generator, and we can turn each $y_{t}^{2}$ into $y_{t+1}^{2}$ by using the formula:

$$
y_{t+1}^{2}=\left(y_{t} y_{t+1}\right)^{2} / y_{t}^{2} \text {. }
$$


In case 2 , we use the fact that $k$ is odd, and therefore the distance from $j$ to $j$ via the cyclic order $y_{j}, y_{j+1}, \ldots, y_{k}, y_{1}, \ldots, y_{j}$ is odd. The telescoping formula will thus yield the desired $y_{j}^{2}$ if we cycle through all the variables $y_{t}$.

The Theorem specifies two types of algebraic bases of size $k$ for $F_{2}\left[y_{1}, \ldots, y_{k}\right]$. To get other bases, we can use the variable transformation $A$ and the mixing transformation $B$ described in Section 2. Due to the invertible and algebraic nature of these transformations, it is easy to translate the representation of each $f$ in the original basis into a representation of $f$ in the new bases.

Example: Consider the algebraic basis $G=\left\{y_{1} y_{2}, y_{2} y_{3}, y_{3} y_{1}\right\}$ of $F_{2}\left[y_{1}, y_{2}, y_{3}\right]$, and the randomly chosen linear transformations:

$$
A=\left(\begin{array}{lll}
37 & 62 & 71 \\
89 & 45 & 68 \\
50 & 17 & 93
\end{array}\right) \quad B=\left(\begin{array}{lll}
41 & 73 & 51 \\
89 & 12 & 60 \\
37 & 94 & 19
\end{array}\right)
$$

Then the linear change of variables $Y \leftarrow A Y(\bmod 101)$ changes the generators $g_{i}$ in $G$ into:

$$
\begin{aligned}
g_{1}^{\prime} & =\left(37 y_{1}+62 y_{2}+71 y_{3}\right)\left(89 y_{1}+45 y_{2}+68 y_{3}\right) \\
& =12 y_{1} y_{2}+38 y_{2} y_{3}+48 y_{3} y_{1}+61 y_{1}^{2}+63 y_{2}^{2}+81 y_{3}^{2} \quad(\bmod 101) \\
g_{2}^{\prime} & =\left(89 y_{1}+45 y_{2}+68 y_{3}\right)\left(50 y_{1}+17 y_{2}+93 y_{3}\right) \\
& =26 y_{1} y 2+89 y_{2} y_{3}+62 y_{3} y_{1}+6 y_{1}^{2}+58 y_{2}^{2}+62 y_{3}^{2} \quad(\bmod 101) \\
g_{3}^{\prime} & =\left(50 y_{1}+17 y_{2}+93 y_{3}\right)\left(37 y_{1}+62 y_{2}+71 y_{3}\right) \\
& =93 y_{1} y_{2}+4 y_{2} y_{3}+22 y_{3} y_{1}+32 y_{1}^{2}+44 y_{2}^{2}+38 y_{3}^{2} \quad(\bmod 101)
\end{aligned}
$$

and the linear transformation $G^{\prime \prime} \leftarrow B G^{\prime}(\bmod 101)$ changes the generators $g_{i}^{\prime}$ in $G^{\prime}$ into the new generators:

$$
\begin{aligned}
g_{1}^{\prime \prime} & =41 g_{1}^{\prime}+73 g_{2}^{\prime}+51 g_{3}^{\prime} \\
& =63 y_{1} y_{2}+78 y_{2} y_{3}+41 y_{3} y_{1}+26 y_{1}^{2}+72 y_{2}^{2}+89 y_{3}^{2} \quad(\bmod 101) \\
g_{2}^{\prime \prime} & =89 g_{1}^{\prime}+12 g_{2}^{\prime}+60 g_{3}^{\prime} \\
& =92 y_{1} y_{2}+44 y_{2} y_{3}+74 y_{3} y_{1}+48 y_{1}^{2}+55 y_{2}^{2}+32 y_{3}^{2} \quad(\bmod 101) \\
g_{3}^{\prime \prime} & =37 g_{1}^{\prime}+94 g_{2}^{\prime}+19 g_{3}^{\prime} \\
& =9 y_{1} y_{2}+51 y_{2} y_{3}+43 y_{3} y_{1}+96 y_{1}^{2}+34 y_{2}^{2}+53 y_{3}^{2} \quad(\bmod 101)
\end{aligned}
$$

Since $G^{\prime \prime}$ is an algebraic basis, it can be used to represent any given quadratic polynomial such as $f=y_{1}^{2}$, but the representation is not obvious unless the invertible transformations $A$ and $B$ are known. 
To simplify our notation, we assume without loss of generality that $k>1$ is odd and $G$ is always the symmetric basis $\left\{y_{i} y_{i+1}\right\}$ (where $i+1$ is computed mod $k)$. Given the invertible linear transformation $Y \leftarrow A Y$, we define $A \& A$ as the $k \times k(k+1) / 2$ matrix whose $i$-th row represents the quadratic polynomial $y_{i} y_{i+1}$ after the change of variables. The coefficients of the final basis $G^{\prime \prime}$ can thus be compactly represented by the $k \times k(k+1) / 2$ matrix $B(A \& A)$.

Assume now that we are given an arbitrary assignment of values $x_{1}, \ldots, x_{k}$ to the elementary quadratics $y_{1} y_{2}, y_{2} y_{3}, \ldots, y_{k} y_{1}$ in the standard basis of $F_{2}\left[y_{1}, \ldots\right.$, $\left.y_{k}\right]$. We can use the telescoping formulas to compute the values of all the $k(k+$ 1)/2 elementary quadratics $y_{i} y_{j}$ for $i \leq j$. We denote this extended version of $X$ by $E(X)$, and note that it increases the height of the column vector from $k$ to $k(k+1) / 2$. The values of the $k$ generators in $G^{\prime \prime}$ for this assignment $X$ can be computed by the matrix-vector product $V=[B(A \& A)][E(X)]$. Note again the non-linearity of this transformation, which is due to the quadratic computation of the coefficients of $A \& A$ from the coefficients of $A$, and the multiplications and divisions required to extend $X$ into $E(X)$.

This function maps the $k$-vector $X$ into the $k$-vector $V$. Our goal now is to invert this function and recover the original values in $X$ when $A$ and $B$ are known. First, we can undo the effect of $B$ by computing $W=B^{-1} V$, and obtain the relationship $W=[A \& A][E(X)]$. By definition, the values $w_{i}$ in $W$ are the values of the generators $g_{i}^{\prime}$ in the intermediate $G^{\prime}$. Since $G^{\prime}$ is an algebraic basis, it can represent any quadratic polynomial in $F_{2}\left[y_{1}, \ldots, y_{k}\right]$ as an algebraic expression in the generators $g_{i}^{\prime}$. In particular it can represent the $k$ elementary quadratics $y_{i} y_{i+1}$ in such a way. However, each $x_{i}$ was defined as the value of $y_{i} y_{i+1}$, and thus it can be recovered by evaluating an appropriate algebraic expression in the values $w_{1}, w_{2}, \ldots, w_{k}$. It is easy to check that these algebraic expressions can be compactly represented as $X=\left[A^{-1} \& A^{-1}\right]\left[E\left(B^{-1} V\right)\right]$.

Example (continued): Consider the algebraic bases $G, G^{\prime}$ and $G^{\prime \prime}$ of the previous example. Let $x_{i}$ denote the value of $y_{i} y_{i+1}$, i.e.: $y_{1} y_{2}=x_{1}, y_{2} y_{3}=$ $x_{2}, \quad y_{3} y_{1}=x_{3} \quad(\bmod 101)$. The values of the other three elementary quadratics can be expressed by as $y_{1}^{2}=x_{3} x_{1} / x_{2}, \quad y_{2}^{2}=x_{1} x_{2} / x_{3}, \quad y_{3}^{2}=x_{2} x_{3} / x_{1}$ $(\bmod 101)$. The values $v_{i}$ of the $g_{i}^{\prime \prime}$ are computed via $[B(A \& A)][E(X)]$, i.e.:

$$
\begin{aligned}
& v_{1}=63 x_{1}+78 x_{2}+41 x_{3}+26 x_{3} x_{1} / x_{2}+72 x_{1} x_{2} / x_{3}+89 x_{2} x_{3} / x_{1} \quad(\bmod 101) \\
& v_{2}=92 x_{1}+44 x_{2}+74 x_{3}+48 x_{3} x_{1} / x_{2}+55 x_{1} x_{2} / x_{3}+32 x_{2} x_{3} / x_{1} \quad(\bmod 101) \\
& v_{3}=9 x_{1}+51 x_{2}+43 x_{3}+96 x_{3} x_{1} / x_{2}+34 x_{1} x_{2} / x_{3}+53 x_{2} x_{3} / x_{1} \quad(\bmod 101)
\end{aligned}
$$

In particular, when the input is $x_{1}=1, x_{2}=2, x_{3}=3$, the output is $v_{1}=$ $54, v_{2}=63, v_{3}=85$. To invert this mapping, we reverse the transformation $G^{\prime \prime} \leftarrow B G^{\prime}(\bmod 101)$ by computing $W=B^{-1} V(\bmod 101)$. When $v_{1}=54$, 
$v_{2}=63, v_{3}=85$, this matrix-vector product yields $w_{1}=94, w_{2}=69, w_{3}=1$, which are the values of the intermediate generators $g_{1}^{\prime}, g_{2}^{\prime}, g_{3}^{\prime}$. We then extend this $W$ into $E(W)=\left(w_{1}, w_{2}, w_{3}, w_{3} w_{1} / w_{2}, w_{1} w_{2} / w_{3}, w_{2} w_{3} / w_{1}\right)$, and compute $X=\left[A^{-1} \& A^{-1}\right][E(W)](\bmod n)$, i.e.:

$$
\begin{aligned}
& x_{1}=29 w_{1}+75 w_{2}+45 w_{3}+74 w_{3} w_{1} / w_{2}+42 w_{1} w_{2} / w_{3}+45 w_{2} w_{3} / w_{1}(\bmod 101) \\
& x_{2}=46 w_{1}+72 w_{2}+14 w_{3}+99 w_{3} w_{1} / w_{2}+61 w_{1} w_{2} / w_{3}+58 w_{2} w_{3} / w_{1}(\bmod 101) \\
& x_{3}=1 w_{1}+58 w_{2}+46 w_{3}+87 w_{3} w_{1} / w_{2}+31 w_{1} w_{2} / w_{3}+77 w_{2} w_{3} / w_{1}(\bmod 101)
\end{aligned}
$$

For $w_{1}=94, w_{2}=69, w_{3}=1$, the extension of $W$ to $E(W)$ yields the column vector $(94,69,1,16,22,19)^{t}$. When these values are substituted into the expressions above, we get the original inputs $x_{1}=1, x_{2}=2, x_{3}=3$.

Note that the application and inversion of the function have essentially the same form. They use a small number of modular additions multiplications and divisions but no root extractions.

The new signature scheme can now be formalized in the following way:

Key Generation: Pick a set $F$ of rational functions in $k$ variables, and a standard algebraic basis $G$ of $F$ with the property that the representation of any $f$ in $F$ as an algebraic expression in terms of the generators $g_{i}$ in $G$ can be easily computed. Transform the easy basis $G$ into an apparently hard basis $G^{\prime \prime}$ by using randomly chosen invertible algebraic transformations. Eliminate $s>0$ generators from $G^{\prime \prime}$, publish the other $k-s$ generators in $G^{\prime \prime}$ as the public key, and keep the algebraic transformations as the private key.

Signature Generation: To sign a given message $m$, assign to each published $g_{i}^{\prime \prime}$ the hashed value $v_{i}=h(m, i)$ of $m$, and to each eliminated $g_{i}^{\prime \prime}$ a newly chosen random number $r_{i}$. Use the secret algebraic transformations to express each $g_{i}$ in the easy basis $G$ in terms of the generators $g_{j}^{\prime \prime}$ in the hard basis $G^{\prime \prime}$. The values $x_{i}$ of the easy generators $g_{i}$ for this assignment of values to the hard generators $g_{j}^{\prime \prime}$ form the signature $X$ of $m$.

Signature Verification: Assign the values $x_{i}$ from the signature $X$ to the easy generators $g_{i}$, and compute the values $v_{i}$ of the $k-s$ hard generators $g^{\prime \prime} i$ which appear in the signer's public key. Evaluate the $k-s$ hashed forms $h(m, i)$ of $m$, and accept the validity of the signature if $v_{i}=h(m, i)$ for all the $k-s$ values of $i$.

The recommended choice for $F$ is the set $F_{d}\left[y_{1}, \ldots, y_{k}\right]$ of all the homogeneous polynomials of degree $d$ over the ring $Z_{n}$. The factorization of $n=p q$ can be destroyed as soon as $n$ is chosen and published. The recommended choice for $G$ is some set of monomials $y_{1}^{e_{1}} y_{2}^{e_{2}} \ldots y_{k}^{e_{k}}$ with $e_{1}+e_{2}+\ldots+e_{k}=d$ such that any other monomial in $F_{\mathrm{d}}\left[y_{1}, \ldots, y_{k}\right]$ can be generated by a sequence of 
multiplications and divisions. It is not difficult to show that for any $d$ and $k$, $F_{d}\left[y_{1}, \ldots, y_{k}\right]$ has an algebraic basis of this type which consists of exactly $k$ monomials of degree $d$ (for example, $G=\left\{y_{1}^{3}, y_{2}^{2} y_{3}, y_{1} y_{2} y_{3}\right\}$ is an algebraic basis for $\left.F_{3}\left[y_{1}, y_{2}, y_{3}\right]\right)$. The problem with large choices of $k$ and $d$ is that the number of coefficients in the published generators $G^{\prime \prime}$ grows as $O\left(k^{d+1}\right)$. However, for fixed $d$ this key size grows only polynomially in $k$, and the recommended choice of $d$ is 2. The recommended bases for $d=2$ are the standard bases $G=$ $\left\{y_{1} y_{2}, \ldots, y_{k-1} y_{k}, y_{k} y_{1}\right\}$ for odd $k$ and $G=\left\{y_{1}^{2}, y_{1} y_{2}, \ldots, y_{k-1} y_{k}\right\}$ for arbitrary $k$. The recommended choice of invertible algebraic transformations is a pair of randomly chosen $k \times k$ matrices $A$ (the variable transformation) and $B$ (the mixing transformation). Note that bases $G$ with $\operatorname{GCD}\left(g_{1}, g_{2}, \ldots, g_{k}\right) \neq 1$ should be avoided - such a GCD remains nontrivial under the transformations $Y-$ $A Y$ and $G^{\prime \prime} \leftarrow B G^{\prime}$, and its computation can reveal some of the rows of $A$. This condition is automatically satisfied in our standard bases $G$ for $k \geq 3$. For example, when $k=3$, two random linear combinations of $y_{1} y_{2}, y_{2} y_{3}$ and $y_{3} y_{1}$ will almost surely be relatively prime. This signature scheme is comparable in its key size, signature size, and computational complexity to the signature scheme described in Section 2. By using the techniques of Shamir[1993], we can show that recovering the secret matrices $A$ and $B$ from the public key $B(A \& A)$ is at least as hard as factoring the modulus $n$ by using the symmetry of the problem when the rows of $A$ and the coloumns of $B$ are cyclically rotated. However, the attack of Coppersmith Stern and Vaudenay overcomes this obstacle by computing signatures directly without extracting the original secret keys.

\section{Bibliography}

1. D. Coppersmith J. Stern and S. Vaudenay [1993]: "Attacks on the Birational Permutation Signature Schemes", Proceedings of CRYPTO 93 (this volume).

2. W. Diffie and M. Hellman [1976]: "New Directions in Cryptography", IEEE Trans. Information Theory, Vol IT-22, No 6, pp 644-654.

3. DSS [1991]: "Specifications for a Digital Signature Standard", US Federal Register Vol 56 No 169, August 301991.

4. H. Fell and W. Diffie [1985]: "Analysis of a Public Key Approach Based on Polynomial Substitution", Proceedings of CRYPTO 85, SpringerVerlag Vol 218, pp 340-349.

5. A. Fiat and A. Shamir [1986]: "How to Prove Yourself: Practical Solutions to Identification and Signature Problems", Proceedings of CRYPTO 86, Springer-Verlag Vol 263, pp 186-194. 
6. R. Merkle and M. Hellman [1978]: "Hiding Information and Signatures in Trapdoor Knapsacks", IEEE Trans. Information Theory, Vol IT-24, No 5, pp 525-530.

7. R. Rivest, A. Shamir and L. Adleman [1978]: "A Method for Obtaining Digital Signatures and Public Key Cryptosystems", Comm. ACM, Vol 21. No 2, pp 120-126.

8. A. Shamir [1993]: "On the Generation of Multivariate Polynomials Which Are Hard To Factor", Proceedings of STOC 1993. 